\title{
Corpus Callosum Atrophy and Cognitive Decline in Early Alzheimer's Disease: Longitudinal MRI Study
}

\author{
Minwei Zhu ${ }^{a} \quad$ Xudong Wang ${ }^{b}$ Wenpeng Gao ${ }^{c}$ Chen Shid Haitao Ge ${ }^{a}$ \\ Hong Shen $^{a}$ Zhiguo Lin ${ }^{a}$ \\ Departments of a Neurosurgery and ${ }^{b}$ Neurology, The First Affiliated Hospital of \\ Harbin Medical University, and 'Bio-X Center, Harbin Institute of Technology, \\ Harbin, PR China; ${ }^{\mathrm{d}}$ New York University Langone Medical Center and School of Medicine, \\ New York, N.Y., USA
}

\author{
Key Words \\ Corpus callosum · Magnetic resonance imaging · Atrophy · Alzheimer's disease · Cognitive \\ decline
}

\begin{abstract}
Background: We investigated the rate of corpus callosum (CC) atrophy and its association with cognitive decline in early Alzheimer's disease (AD). Methods: We used publicly available longitudinal MRI data corresponding to 2 or more visits from 137 subjects characterized using the Clinical Dementia Rating (CDR) score. We classified these subjects into 3 groups according to the progression of their cognitive status: a healthy control group (CDR $0 \rightarrow 0, n=$ 72 ), a decliner group (CDR $0 \rightarrow 0.5, \mathrm{n}=14$ ) and an AD group (CDR $0.5 \rightarrow 0.5 / 1, \mathrm{n}=51$ ). We measured the CC area on the midsagittal plane and calculated the atrophy rate between 2 or more visits. The correlation between the CC atrophy rate and annualized Mini Mental State Examination (MMSE) change was also analyzed. Results: The results indicated that the baseline CC area was larger in the healthy control group compared to the AD group, whereas the CC atrophy rate was higher in the $A D$ group relative to the control and decliner groups. The CC atrophy rate was also correlated with the annualized MMSE change in AD patients $(p<0.05)$. Conclusion: Callosal atrophy is present even in early $A D$ and subsequently accelerates, such that the rate of $C C$ atrophy is associated with cognitive decline in $A D$ patients.
\end{abstract}


Dementia

Cognitive Disorders

\begin{tabular}{l|l}
\hline \multicolumn{2}{l}{ Dement Geriatr Cogn Disord 2014;37:214-222 } \\
\hline DOI: $\underline{10.1159 / 000350410}$ & $\begin{array}{l}\text { C 2013 S. Karger AG, Basel } \\
\text { www.karger.com/dem }\end{array}$ \\
\hline
\end{tabular}

Zhu et al.: Corpus Callosum Atrophy and Cognitive Decline in Early Alzheimer's Disease: Longitudinal MRI Study

\section{Introduction}

Alzheimer's disease (AD) is an irreversible, neurodegenerative brain disease characterized by progressive cognitive decline. The effects of AD on gray matter have been repeatedly demonstrated, although several investigations have shown that AD is also associated with white matter [1-4]. The corpus callosum (CC), as the largest white matter fiber bundle in the brain, is particularly susceptible to atrophy in AD [5-7].

Magnetic resonance imaging (MRI) has been widely used to assess CC atrophy in vivo, and most cross-sectional studies have documented that patients with AD exhibit CC atrophy [5-15]. Although not all reports are in agreement, patterns of atrophy in the anterior and posterior subregions of the CC, as well as in the total CC area, are the most consistent findings in AD patients [5, 6, 16-18]. Prior work has proposed diverse mechanisms for CC atrophy in AD [5, $6,19]$. For example, the Wallerian degeneration hypothesis suggests that the death of projecting pyramidal cells in the neocortex may contribute to the atrophy of the posterior $\mathrm{CC}$, while the myelin breakdown hypothesis has been proposed to explain the atrophy of the anterior CC.

Elderly adults with mild cognitive deficits can progress very differently as they age; some may remain stable, but these individuals also have a high risk of developing AD. It is unclear whether cognitive changes during aging reflect CC atrophy.

Most previous studies [5-15] have focused on the investigation of CC atrophy using cross-sectional data, in which intersubject variance is inevitable. However, the analysis of longitudinal data can reduce the impact of intersubject variance by measuring changes within individuals relative to their own baselines, and this type of analysis can also control for differences associated with cross-sectional data. For instance, cross-sectional samples may include hidden group heterogeneity (cohort effects), such as differences in the living environments of the patients. However, longitudinal data may reduce this heterogeneity, as they are obtained by repeated imaging of the same subject over time [20]. To the best of our knowledge, only one study has investigated longitudinal cognitive decline in relation to CC atrophy in AD patients [10], and no study to date has applied the Clinical Dementia Rating (CDR) scale [21] to early-stage $\mathrm{AD}$ patients. The $\mathrm{CDR}$ is a numeric scale used to rate the severity of symptoms of dementia, where a CDR of 0 represents no dementia and CDRs of $0.5,1,2$, and 3 indicate very mild, mild, moderate, and severe dementia, respectively.

Early diagnosis and intervention in patients with $\mathrm{AD}$ contribute to slow disease progression, which lends considerable interest to the identification of reliable biomarkers [22]. In this paper, we measured the area of a section of the CC at multiple time points, which led us to determine the rate of CC atrophy in initially nondemented patients who converted to dementia (CDR $0 \rightarrow 0.5$ ) and AD (CDR $0.5 \rightarrow 0.5 / 1$ ). We also explored whether CC area and $\mathrm{CC}$ atrophy rate were correlated with cognitive decline.

\section{Materials and Methods}

\section{Subjects and Imaging Data}

The data analyzed in this study were obtained from the Open Access Series of Imaging Studies (OASIS) database. This database provides a repository of freely available MRI data to the scientific community to encourage continued research on aging and disease processes [23]. The present longitudinal MRI data included 150 subjects aged 60-96 years. For each subject, T1-weighted magnetization-prepared rapid gradient-echo images were obtained using a 1.5-tesla scanner (Siemens Healthcare, Erlangen, Germany) with a repetition time of $9.7 \mathrm{~ms}$, echo time of $4 \mathrm{~ms}$, flip angle of $10^{\circ}$, inversion time of $20 \mathrm{~ms}$, delay time of $200 \mathrm{~ms}$ and resolution of $256 \times 256(1 \times 1 \mathrm{~mm})$.

The OASIS database also provided information on the subjects concerning their results from the CDR and Mini Mental State Examination (MMSE) at the initial visit and subsequent visits, as well as their estimated 
Zhu et al.: Corpus Callosum Atrophy and Cognitive Decline in Early Alzheimer's Disease: Longitudinal MRI Study
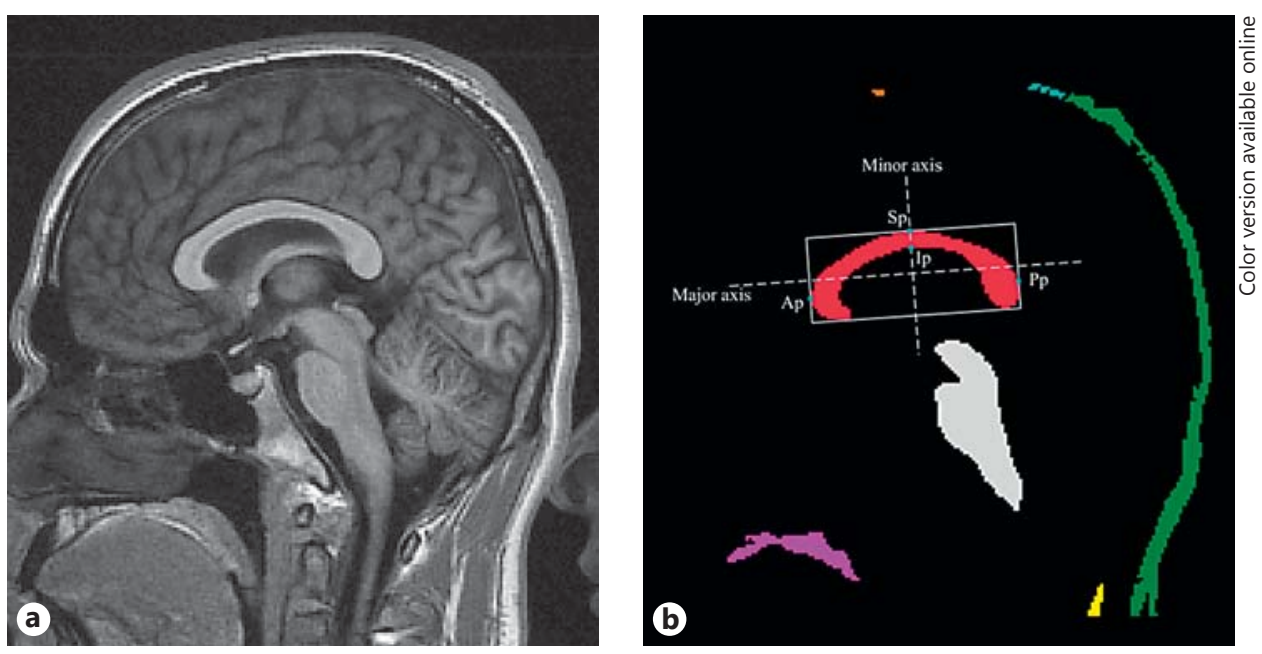

Fig. 1. Procedure for segmenting the CC. a MR image on the MSP extracted from the MR volume. b Region of interest of the CC obtained with dual-threshold segmentation. Ap (Pp) = The most anterior (posterior) point of the CC on the major axis; Sp (Ip) = the most superior (inferior) point of the CC on the minor axis.

total intracranial volume (eTIV). The MMSE was used to assess general cognitive function, while the eTIV was used to assess brain size. Subjects with dementia not caused by AD (e.g. vascular dementia) were excluded. Additional details about the database have previously been described by Marcus et al. [24].

The diagnosis of AD was based on clinical information (derived mainly from a collateral source) indicating that the participant had experienced gradual and progressive impairment in memory and other cognitive domains. The collateral source and participant interview yield a global CDR score, and individuals with a CDR of 0.5 or greater were diagnosed with AD based on standard criteria, which have been validated by histopathological examination [25], and subjects with a CDR of 0.5 were classified in the earliest symptomatic stage of AD.

Because the focus of this study was on the early stage of AD, we excluded 13 subjects with an initial CDR of 1 , which left 137 subjects with a CDR of 0 (86 subjects) or 0.5 (51 subjects) at their initial visit. Fifty of these subjects were reexamined after 1 year, 27 after 2 years and the remaining 60 subjects after 3 years. We classified the subjects into 3 groups based on the trajectory of their CDR results. The 14 initially healthy subjects who progressed to $\mathrm{AD}$ at the second visit (CDR $0 \rightarrow 0.5$ ) were classified as the decliner group; the 72 subjects who remained stable (CDR $0 \rightarrow 0$ ) were classified as the healthy control group; and the remaining 51 subjects who showed symptoms of very mild dementia at the initial visit were classified as the AD group (CDR $0.5 \rightarrow 0.5 / 1)$.

\section{Measurement of CC Size}

The CC on the midsagittal plane (MSP) was segmented using a previously described semi-automatic method [15]. The method consists of the following 3 steps:

Step 1: Extraction of the MSP from the MR volume (fig. 1a);

Step 2: Dual thresholding-based segmentation;

Step 3: Selection of the CC region by identifying the callosal shape (fig. 1b).

Due to the anatomical variability and the artifacts in MR images, mis- or over-segmentation may occur in some cases. Thus, 2 raters were tasked to amend these flaws mutually and manually with the NASP [26], which also provided manual delineation. Finally, the area of the CC was calculated by multiplying the number of pixels by the pixel size.

\section{Quantification of CC Atrophy and Cognitive Decline}

We quantified the rate of CC atrophy in each subject as the slope of the line connecting the first and second measurements of CC area. We normalized each such slope for intersubject variance by dividing it by the CC area measured on the first MRI scan. For a subject scanned twice at an interval of t years, the CC 


\begin{tabular}{l|l}
\hline DOI: $10.1159 / 000350410$ & $\begin{array}{l}\text { C) } 2013 \text { S. Karger AG, Basel } \\
\text { www.karger.com/dem }\end{array}$ \\
\hline $\begin{array}{l}\text { Zhu et al.: Corpus Callosum Atrophy and Cognitive Decline in Early Alzheimer's } \\
\text { Disease: Longitudinal MRI Study }\end{array}$
\end{tabular}

Table 1. Demographic or clinical variables for each group

\begin{tabular}{lccc}
\hline Descriptive variable & $\begin{array}{l}\text { Healthy control group } \\
(\text { CDR 0 } \rightarrow 0)\end{array}$ & $\begin{array}{l}\text { Decliner group } \\
\text { (CDR 0 } \rightarrow 0.5)\end{array}$ & $\begin{array}{c}\text { AD group } \\
\text { (CDR 0.5 } \rightarrow 0.5 / 1)\end{array}$ \\
\hline Patients, $\mathrm{n}$ & 72 & 14 & 51 \\
Age baseline, years & $75.43 \pm 8.23$ & $77.07 \pm 7.69$ & $74.96 \pm 6.23$ \\
Men/women & $22 / 50^{\mathrm{a}}$ & $4 / 10$ & $30 / 21^{\mathrm{a}}$ \\
Education, years & $15.26 \pm 2.60^{\mathrm{a}}$ & $15.15 \pm 2.57$ & $13.61 \pm 2.86^{\mathrm{a}}$ \\
eTIV, cm $^{3}$ & $1,480.39 \pm 183.78$ & $1,483.29 \pm 132.95$ & $1,463.06 \pm 168.22$ \\
MMSE baseline $_{\text {Annualized MMSE change }}^{29.19 \pm 0.85^{\mathrm{a}}}$ & $29.36 \pm 0.93^{\mathrm{b}}$ & $25.92 \pm 3.09^{\mathrm{a}, \mathrm{b}}$ \\
CC area baseline, mm & & $-0.59 \pm 0.86$ & $-0.62 \pm 1.54^{\mathrm{a}}$ \\
CC atrophy rate, \%/year & $-0.03 \pm 0.62^{\mathrm{a}}$ & $560.36 \pm 61.43$ & $542.96 \pm 67.95^{\mathrm{a}}$ \\
\hline
\end{tabular}

Data are expressed as the mean \pm standard deviation or as counts.

a, b Identical superscripts indicate statistically significant pairwise differences $(p<0.05)$.

atrophy rate was calculated as $\left(A_{2}-A_{1}\right) / A_{1} t$, where $A_{1}$ and $A_{2}$ represent the CC area measured on the first and second MRI scans, respectively. We quantified cognitive decline in a similar fashion by calculating the annual change in MMSE between the 2 observations.

\section{Statistical Analysis}

Statistical analysis was performed using SPSS (Release 13.0, The LEAD Technologies Inc., Chicago, Ill., USA). The gender ratios of the 3 groups were compared using $\chi^{2}$ tests. Continuous variables (age, education, eTIV, MMSE baseline, annualized MMSE change, CC atrophy rate) were compared using an analysis of variance (ANOVA), and we then used an analysis of covariance (ANCOVA) to compare the CC area at baseline and the CC atrophy rate across groups, with age and gender as covariates. Post hoc comparisons were performed using the Bonferroni test. Associations of baseline CC area and CC atrophy rate with baseline MMSE and annualized MMSE change were assessed using partial correlations, corrected for age and gender. Scatterplots of baseline CC area and CC atrophy rate versus annualized change in MMSE were constructed. A p value $<0.05$ was considered significant.

\section{Results}

\section{Inter- and Intra-Rater Reliability}

To determine the inter- and intra-rater agreements of the measurements, 2 experts segmented the CC in MR scans of 40 subjects with the NASP. Each expert segmented the CC twice in a 2-week period to avoid previous bias. Inter- and intra-rater reliabilities were quantified by Pearson's correlation across raters and across measurements within each rater, respectively. Inter-rater reliability was very high, ranging from $0.931(\mathrm{p}<0.001)$ to 0.954 $(\mathrm{p}<0.001)$, and intra-rater reliability was also high, with correlations ranging from 0.956 $(\mathrm{p}<0.001)$ to $0.989(\mathrm{p}<0.001)$. These values indicate that the inter- and intra-rater variation of our method was low.

\section{Comparison of Demographic Descriptive Characteristics between the Groups}

The basic descriptive characteristics of the patients are listed in table 1 . The 3 groups did not differ in age, baseline values, or eTIV ( $p>0.05$ ). However, the control and AD groups differed in terms of gender ratio $(p<0.01)$ and education $(p<0.05)$, and the MMSE baseline in the AD group was lower than that in the control and decliner groups $(\mathrm{p}<0.05)$, as expected. 
Zhu et al.: Corpus Callosum Atrophy and Cognitive Decline in Early Alzheimer's Disease: Longitudinal MRI Study

Fig. 2. a Bar graph illustrating baseline CC areas in the healthy control (CDR $0 \rightarrow 0$ ), decliner (CDR $0 \rightarrow 0.5$ ) and AD (CDR $0.5 \rightarrow 0.5 / 1$ ) groups. b Bar graph illustrating CC atrophy rates in the healthy control (CDR $0 \rightarrow 0$ ), decliner (CDR $0 \rightarrow 0.5$ ) and AD (CDR $0.5 \rightarrow 0.5 / 1$ ) groups. Error bars represent the standard deviation. Differences between groups were assessed using ANOVA. * Significant difference at $\mathrm{p}<0.05$.
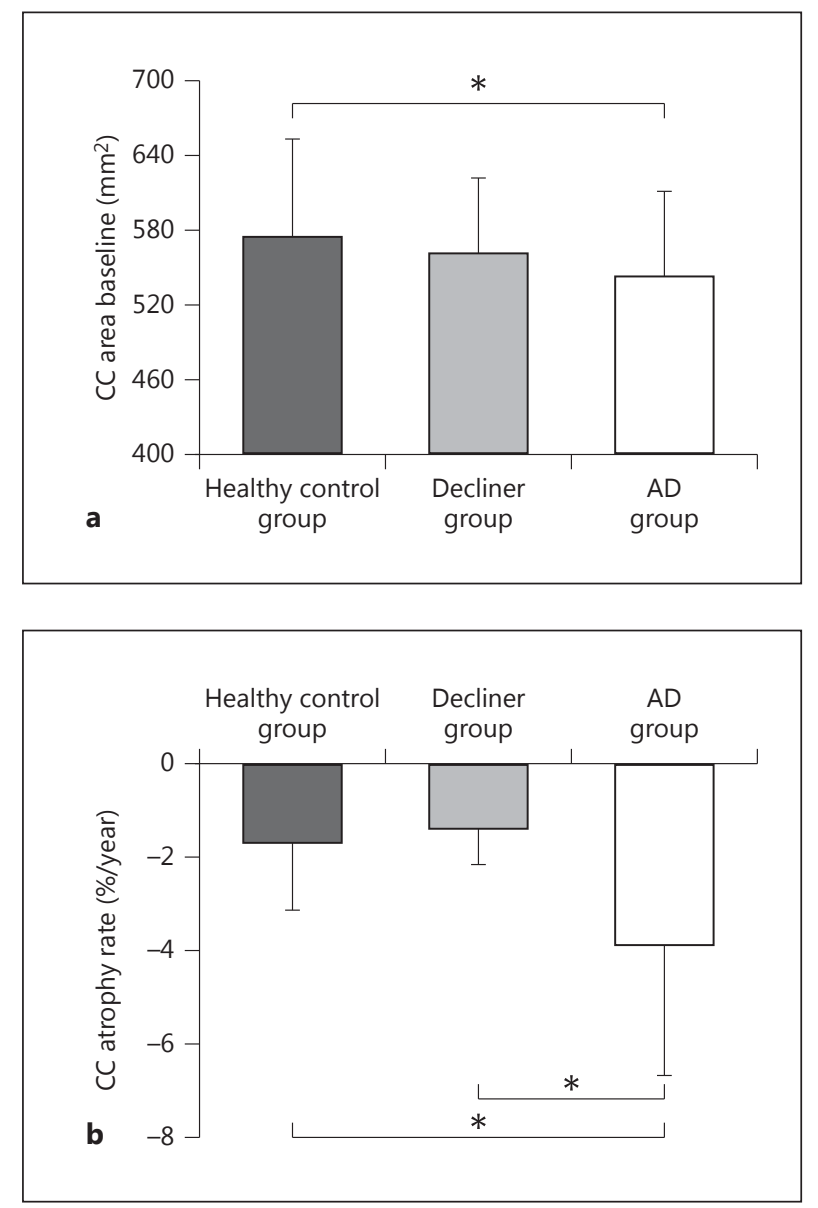

\section{Area Baseline and CC Atrophy Rate}

We observed group differences in both baseline CC area and CC atrophy rate $(\mathrm{p}<0.05$; table 1). Post hoc analysis indicated that the CC area at baseline in the AD group was smaller in comparison to the healthy control group (fig. 2a) but was not significantly different in comparison to the decliner group ( $p>0.05$ ). In addition, the AD group demonstrated a higher $\mathrm{CC}$ atrophy rate compared to the healthy control and decliner groups (fig. 2b), but the healthy control and decliner groups did not differ in this measure $(\mathrm{p}>0.05)$.

\section{Cognitive Decline}

To investigate whether baseline CC area and CC atrophy rate reflected cognitive decline, we evaluated association with the baseline MMSE and the annualized MMSE change. Partial correlation analysis demonstrated that baseline CC area was not associated with either baseline MMSE $(r=0.014, p=0.875)$ or annualized change in MMSE $(r=0.055, p=0.524$; fig. 3). In contrast, CC atrophy rate was associated with both baseline MMSE $(r=0.184, p<$ $0.05)$ and the change in MMSE $(r=0.236, p<0.01)$. Correlations computed within the 3 groups indicated no association between CC atrophy rate and baseline MMSE in any group. However, the CC atrophy rate within the AD group was associated with annualized MMSE change $(r=0.318, p<0.05)$, while no such association was found in the healthy control group $(\mathrm{r}=0.157, \mathrm{p}=0.188)$ or the decliner group $(\mathrm{r}=0.628, \mathrm{p}=0.125)$. 
Zhu et al.: Corpus Callosum Atrophy and Cognitive Decline in Early Alzheimer's Disease: Longitudinal MRI Study

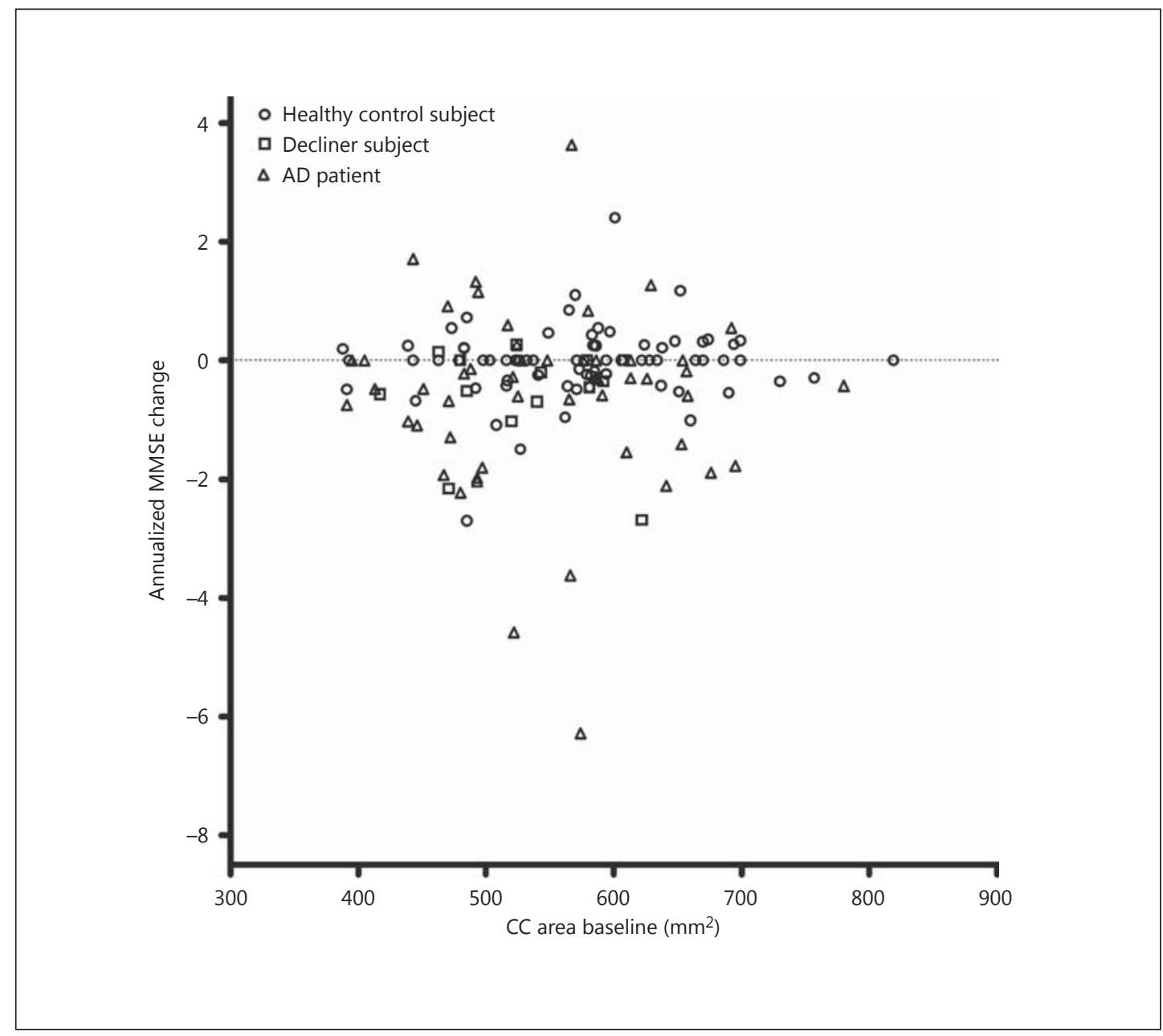

Fig. 3. Scatterplots of CC area baseline according to annual change in MMSE.

\section{Discussion}

The CC connects the hemispheres of the brain and plays a key role in the integration of perception and action. It has been well established that the CC is involved in high-order cognitive functions [27] and that CC atrophy contributes to cognitive impairment in AD patients. To the best of our knowledge, few studies have investigated the relationship between CC atrophy and cognitive decline in the early stage of $\mathrm{AD}[10,20]$. In this paper, we estimated the CC atrophy rate in our subjects using MRI-based measures, quantified their cognitive decline with annualized MMSE change, and analyzed the correlation between these measures of brain and cognitive health.

We found that baseline CC area in the AD group was significantly smaller than that in the healthy control group but statistically indistinguishable from the decliner group. Thus, subjects with normal mental status (CDR 0) had larger CC areas than those in the AD group, who suffered from very mild dementia (CDR 0.5 ). Most studies on this topic have found smaller baseline CC areas in $\mathrm{AD}$ patients compared to healthy controls. According to recent findings reviewed by Di Paola et al. [19], both Wallerian degeneration and myelin breakdown are responsible for callosal atrophy in early-stage AD patients. Wallerian degeneration mainly affects the posterior 
Zhu et al.: Corpus Callosum Atrophy and Cognitive Decline in Early Alzheimer's Disease: Longitudinal MRI Study

region of the CC, whereas myelin breakdown is often present in the anterior region. Our findings add further support for the presence of CC atrophy even in early stages of dementia.

We found that the CC atrophy rate of the AD group was significantly higher than that of the healthy control and decliner groups, which did not differ from one another. This finding is somewhat similar to that of the study of Teipel et al. [10], although these authors reported different magnitudes of annualized CC atrophy (roughly 7.7\% in the AD group and $0.9 \%$ in the healthy control group, as compared to our observations of 3.86 and $1.69 \%$, respectively). This discrepancy may have been due to different classification rules; Teipel et al. [10] included $\mathrm{AD}$ patients whose symptoms ranged from mild to severe, whereas our AD group included patients with very mild symptoms (CDR $0.5 \rightarrow 0.5 / 1$ ), and our study mainly focused on a homogeneous group of patients. We did not find significant differences in the rate of CC atrophy between the healthy control and decliner groups, which suggests that patients progress from being healthy to having mild dementia without significant acceleration in the rate of CC atrophy relative to controls. However, once patients meet the criteria for dementia, CC atrophy accelerates significantly.

The current study also investigated the association between CC atrophy and annualized MMSE change in the early stage of AD. These 2 measures were significantly correlated in the $\mathrm{AD}$ group, indicating that the rate of $\mathrm{CC}$ atrophy in $\mathrm{AD}$ patients is associated with their rate of cognitive decline.

We did not find a significant correlation between annualized MMSE score change and baseline CC area in any of the groups, which is inconsistent with the results of a recent study [28] showing that smaller CC area was correlated with more severe decline in MMSE score. This discrepancy may have been due to differences in aspects of experimental design, such as numbers of subjects and classification criteria for AD patients. Frederiksen et al. [28] classified AD patients using the fourth edition of the Diagnostic and Statistical Manual of Mental Disorders (DSM-IV) and McKhann's criteria, while our study classified patients according to their CDR score. In addition, our study included a relatively large number of subjects compared to this previous study, which may have increased our statistical power.

There are several traditional methods for parcellating the CC according to its geometric features, which have been previously described by Witelson [29], Hensel et al. [30], Weis et al. [31] and Hampel et al. [13]. However, more recent studies based on these schemes have generated controversial results concerning the assumed topography of the callosal fiber tracts $[9,32]$. Therefore, we did not subdivide the CC into subregions in this study. Our study was also limited by the small number of subjects in the decliner group relative to the other 2 groups. As additional longitudinal data become available, a more sensitive investigation into CC change during early AD will become possible.

In conclusion, our study provides important results that greatly improve our understanding of the role of the CC in the early stage of AD; in particular, we found that accelerated $\mathrm{CC}$ atrophy was associated with cognitive decline. Because AD patients have a higher rate of CC atrophy, measurements of CC area from repeat MRI analyses could be helpful for generating diagnostic work-ups for patients who may suffer from dementia, and these measurements may also inform evaluations of the efficacy of pharmacological interventions in AD patients.

\section{Acknowledgments}

The OASIS data used in this study were collected as part of the following grants: P50 AG05681, P01 AG03991, R01 AG021910, P50 MH071616, U24 RR021382 and R01 MH56884. We especially thank the Washington University Alzheimer's Disease Research Center for making the MRI data available. 
Zhu et al.: Corpus Callosum Atrophy and Cognitive Decline in Early Alzheimer's Disease: Longitudinal MRI Study

This work was supported by grants from the National Natural Science Foundation of China (No. 81201150, No. 81071219, No. 81171304 and No. 81071215) as well as by Fundamental Research Funds for the Central Universities (Grant No. HIT. NSRIF. 2013106), Heilongjiang Postdoctoral Financial Assistance (LBH-Z11167), the Science Foundation of Heilongjiang Province (D201062), and the Foreign Collaboration Project of Heilongjiang Province (WB10B104).

\section{Disclosure Statement}

The authors declare no conflicts of interest.

\section{References}

1 Hua X, Leow AD, Lee S, Klunder AD, Toga AW, Lepore N, Chou YY, Brun C, Chiang MC, Barysheva M, Jack CR Jr, Bernstein MA, Britson PJ, Ward CP, Whitwell JL, Borowski B, Fleisher AS, Fox NC, Boyes RG, Barnes J, Harvey D, Kornak J, Schuff N, Boreta L, Alexander GE, Weiner MW, Thompson PM, Alzheimer's Disease Neuroimaging Initiative: 3D characterization of brain atrophy in Alzheimer's disease and mild cognitive impairment using tensor-based morphometry. Neuroimage 2008;41:19-34.

2 Li J, Pan P, Huang R, Shang H: A meta-analysis of voxel-based morphometry studies of white matter volume alterations in Alzheimer's disease. Neurosci Biobehav Rev 2012;36:757-763.

3 Smith CD, Snowdon DA, Wang H, Markesbery WR: White matter volumes and periventricular white matter hyperintensities in aging and dementia. Neurology 2000;54:838-842.

- 4 Bronge L, Bogdanovic N, Wahlund LO: Postmortem MRI and histopathology of white matter changes in Alzheimer brains: a quantitative, comparative study. Dement Geriatr Cogn Disord 2002;13:205-212.

5 Di Paola M, Di Iulio F, Cherubini A, Blundo C, Casini AR, Sancesario G, Passafiume D, Caltagirone C, Spalletta G: When, where, and how the corpus callosum changes in MCI and AD: a multimodal MRI study. Neurology 2010; 74:1136-1142.

- 6 Di Paola M, Luders E, Di Iulio F, Cherubini A, Passafiume D, Thompson PM, Caltagirone C, Toga AW, Spalletta G: Callosal atrophy in mild cognitive impairment and Alzheimer's disease: different effects in different stages. Neuroimage 2010;49:141-149.

- 7 Wang PJ, Saykin AJ, Flashman LA, Wishart HA, Rabin LA, Santulli RB, McHugh TL, MacDonald JW, Mamourian AC: Regionally specific atrophy of the corpus callosum in AD, MCI and cognitive complaints. Neurobiol Aging 2006;27:1613-1617.

8 Thomann PA, Wustenberg T, Pantel J, Essig M, Schroder J: Structural changes of the corpus callosum in mild cognitive impairment and Alzheimer's disease. Dement Geriatr Cogn Disord 2006;21:215-220.

- 9 Tomaiuolo F, Scapin M, Di Paola M, Le Nezet P, Fadda L, Musicco M, Caltagirone C, Collins DL: Gross anatomy of the corpus callosum in Alzheimer's disease: regions of degeneration and their neuropsychological correlates. Dement Geriatr Cogn Disord 2007;23:96-103.

-10 Teipel SJ, Bayer W, Alexander GE, Zebuhr Y, Teichberg D, Kulic L, Schapiro MB, Moller HJ, Rapoport SI, Hampel H: Progression of corpus callosum atrophy in Alzheimer disease. Arch Neurol 2002;59:243-248.

-11 Hampel H, Teipel SJ, Alexander GE, Pogarell O, Rapoport SI, Moller HJ: In vivo imaging of region and cell type specific neocortical neurodegeneration in Alzheimer's disease: perspectives of MRI derived corpus callosum measurement for mapping disease progression and effects of therapy - evidence from studies with MRI, EEG and PET. J Neural Transm 2002;109:837-855.

-12 Teipel SJ, Bayer W, Alexander GE, Bokde AL, Zebuhr Y, Teichberg D, Muller-Spahn F, Schapiro MB, Moller HJ, Rapoport SI, Hampel H: Regional pattern of hippocampus and corpus callosum atrophy in Alzheimer's disease in relation to dementia severity: evidence for early neocortical degeneration. Neurobiol Aging 2003;24:85-94.

13 Hampel H, Teipel SJ, Alexander GE, Horwitz B, Teichberg D, Schapiro MB, Rapoport SI: Corpus callosum atrophy is a possible indicator of region- and cell type-specific neuronal degeneration in Alzheimer disease: a magnetic resonance imaging analysis. Arch Neurol 1998;55:193-198.

14 Lyoo IK, Satlin A, Lee CK, Renshaw PF: Regional atrophy of the corpus callosum in subjects with Alzheimer's disease and multi-infarct dementia. Psychiatry Res 1997;74:63-72.

15 Zhu M, Gao W, Wang X, Shi C, Lin Z: Progression of corpus callosum atrophy in early stage of Alzheimer's disease: MRI based study. Acad Radiol 2012;19:512-517.

-16 Wang H, Su MY: Regional pattern of increased water diffusivity in hippocampus and corpus callosum in mild cognitive impairment. Dement Geriatr Cogn Disord 2006;22:223-229.

17 Xie S, Xiao JX, Gong GL, Zang YF, Wang YH, Wu HK, Jiang XX: Voxel-based detection of white matter abnormalities in mild Alzheimer disease. Neurology 2006;66:1845-1849.

18 Wang L, Goldstein FC, Veledar E, Levey AI, Lah JJ, Meltzer CC, Holder CA, Mao H: Alterations in cortical thickness and white matter integrity in mild cognitive impairment measured by whole-brain cortical thickness mapping and diffusion tensor imaging. AJNR Am J Neuroradiol 2009;30:893-899. 
19 Di Paola M, Spalletta G, Caltagirone C: In vivo structural neuroanatomy of corpus callosum in Alzheimer's disease and mild cognitive impairment using different MRI techniques: a review. J Alzheimers Dis 2010;20: 67-95.

20 Hensel A, Wolf H, Busse A, Arendt T, Gertz HJ: Association between global brain volume and the rate of cognitive change in elderly humans without dementia. Dement Geriatr Cogn Disord 2005;19:213-221.

-21 Morris JC: The clinical dementia rating (CDR): current version and scoring rules. Neurology 1993;43:24122414.

22 Mueller SG, Weiner MW, Thal LJ, Petersen RC, Jack CR, Jagust W, Trojanowski JQ, Toga AW, Beckett L: Ways toward an early diagnosis in Alzheimer's disease: the alzheimer's disease neuroimaging initiative (ADNI). Alzheimers Dement 2005;1:55-66.

23 Marcus DS, Wang TH, Parker J, Csernansky JG, Morris JC, Buckner RL: Open access series of imaging studies (OASIS): cross-sectional MRI data in young, middle aged, nondemented, and demented older adults. J Cogn Neurosci 2007;19:1498-1507.

-24 Marcus DS, Fotenos AF, Csernansky JG, Morris JC, Buckner RL: Open access series of imaging studies: longitudinal MRI data in nondemented and demented older adults. J Cogn Neurosci 2010;22:2677-2684.

-25 Berg L, McKeel DW Jr, Miller JP, Storandt M, Rubin EH, Morris JC, Baty J, Coats M, Norton J, Goate AM, Price JL, Gearing M, Mirra SS, Saunders AM: Clinicopathologic studies in cognitively healthy aging and Alzheimer's disease: relation of histologic markers to dementia severity, age, sex, and apolipoprotein E genotype. Arch Neurol 1998;55:326-335.

26 Fu Y, Gao W, Zhu M, Chen X, Lin Z, Wang S: Computer-assisted automatic localization of the human pedunculopontine nucleus in T1-weighted MR images: a preliminary study. Int J Med Robot 2009;5:309-318.

27 Schulte T, Muller-Oehring EM: Contribution of callosal connections to the interhemispheric integration of visuomotor and cognitive processes. Neuropsychol Rev 2010;20:174-190.

-28 Frederiksen KS, Garde E, Skimminge A, Ryberg C, Rostrup E, Baare WF, Siebner HR, Hejl AM, Leffers AM, Waldemar G: Corpus callosum atrophy in patients with mild Alzheimer's disease. Neurodegener Dis 2011;8: 476-482.

29 Witelson SF: Hand and sex differences in the isthmus and genu of the human corpus callosum: a postmortem morphological study. Brain 1989;112(Pt 3):799-835.

-30 Hensel A, Wolf H, Kruggel F, Riedel-Heller SG, Nikolaus C, Arendt T, Gertz HJ: Morphometry of the corpus callosum in patients with questionable and mild dementia. J Neurol Neurosurg Psychiatry 2002;73:59-61.

-31 Weis S, Jellinger K, Wenger E: Morphometry of the corpus callosum in normal aging and Alzheimer's disease. J Neural Transm Suppl 1991;33:35-38.

32 Hofer S, Frahm J: Topography of the human corpus callosum revisited - comprehensive fiber tractography using diffusion tensor magnetic resonance imaging. Neuroimage 2006;32:989-994. 